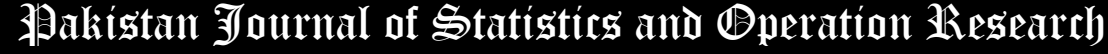

\section{The four-parameter Fréchet distribution: Properties and applications}

\author{
M. S. Hamed ${ }^{1,2}$, Fahad Aldossary ${ }^{3}$, Ahmed Z. Afify ${ }^{4 *}$
}

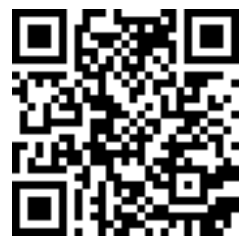

* Corresponding Author

1. Management Information System Department, Taibah University, Saudi Arabia, mshamed@taibahu.edu.sa

2. Department of Statistics, Mathematics and Insurance Benha University, Egypt

3. Management Information System Department, Taibah University, Saudi Arabia, fdossary@taibahu.edu.sa

4. Department of Statistics, Mathematics and Insurance, Benha University, Egypt, ahmed.afify@fcom.bu.edu.eg

\begin{abstract}
In this article, we propose a new four-parameter Fréchet distribution called the odd Lomax Fréchet distribution. The new model can be expressed as a linear mixture of Fréchet densities. We provide some of its mathematical properties. The estimation of the model parameters is performed by the maximum likelihood method. We illustrate the good performance of the maximum likelihood estimates via a detailed numerical simulation study. The importance and usefulness of the proposed distribution for modeling data are illustrated using two real data applications.
\end{abstract}

Key Words: Fréchet distribution; Maximum likelihood estimation; Odd Lomax-G class; Order statistics.

Mathematical Subject Classification: 60E05, 62E15.

\section{Introduction}

The Fréchet distribution (Fréchet, 1924) is one of the important distributions in extreme value theory and it has many applications in accelerated life testing, rainfall, earthquakes, floods, horse racing, wind speeds and sea waves. For more details about the Fréchet distribution and its applications see, e.g., Kotz and Nadarajah (2000) and Mubarak (2011).

There are many generalizations of the Fréchet distribution in the statistical literature. For example, Nadarajah and Kotz (2003) introduced the exponentiated Fréchet, Nadarajah and Gupta (2004) studied the beta Fréchet, Mahmoud and Mandouh (2013) proposed the transmuted Fréchet, Krishna et al. (2013) and Silva et al. (2013) introduced the Marshall-Olkin Fréchet and the gamma extended Fréchet, respectively, Mead and Abd-Eltawab (2014) proposed the Kumaraswamy Fréchet, Afify et al. (2015) and Afify et al. (2016) introduced the transmuted Marshall-Olkin Fréchet and the Weibull Fréchet, respectively, Mead et al. (2017) introduced the Beta Exponential Fréchet, Ramos et al. (2017) introduced a new generalization of the Fréchet distribution with long-term survival, Tablada and Cordeiro (2017) studied the modified Fréchet, Mansour et al. (2018a) and Mansour et al. (2018b) proposed the odd Lindley Fréchet and Kumaraswamy exponentiated Fréchet, respectively and Abouelmagd et al. (2018) introduced the Burr X Fréchet distribution, among others. For more details about the Fréchet distribution and its applications see Ramos et al. (2019). 
The cumulative distribution function $(\mathrm{CDF})$ of the Fréchet distribution is given by (for $x>0$ )

$$
G(x ; a, b)=\exp \left[-\left(\frac{a}{x}\right)^{b}\right]
$$

The corresponding probability density function (PDF) of (1) is

$$
g(x ; a, b)=b a^{b} x^{-b-1} \exp \left[-\left(\frac{a}{x}\right)^{b}\right]
$$

where $a>0$ is a scale parameter and $b>0$ is a shape parameter.

Let $g(x ; \zeta)$ and $G(x ; \zeta)$ denote the PDF and CDF of a baseline model with parameter vector $\zeta$. Cordeiro et al. (2019) proposed a new class of distribution called the Odd Lomax-G (OLX-G) family.

The CDF of the OLx-G family is given by

$$
F(x ; \alpha, \beta, \zeta)=1-\beta^{\alpha}\left[\beta+\frac{G(x ; \zeta)}{1-G(x ; \zeta)}\right]^{-\alpha}
$$

where $\alpha$ and $\beta$ are positive shape parameter.

The OLx-G PDF is

$$
f(x ; \alpha, \beta, \zeta)=\alpha \beta^{\alpha} \frac{g(x ; \zeta)}{[1-G(x ; \zeta)]^{2}}\left[\beta+\frac{G(x ; \zeta)}{1-G(x ; \zeta)}\right]^{-\alpha-1}
$$

where $g(x ; \zeta)=d G(x ; \zeta) / d x$

A random variable $X$ with PDF (4) is denoted by $X \sim \mathrm{OLx}-\mathrm{G}(\alpha, \beta, \zeta)$. The hazard rate function (HRF) of the OLx-G class reduces to

$$
h(x ; \alpha, \beta, \zeta)=\frac{\alpha g(x ; \zeta)}{[1-G(x ; \zeta)]^{2}\left[\beta+\frac{G(x ; \zeta)}{1-G(x ; \zeta)}\right]}
$$

The rest of this article is organized as follows. In Section 2, we define the OLxF distribution and provide some plots for its PDF and hazard rate function (HRF). Section 3 is devoted to the derivation of a useful linear representation for the OLxF PDF. We derive some mathematical properties of the OLxF distribution including ordinary and incomplete moments, moment generating function and order statistics in Section 4. The maximum likelihood estimates of the OLxF parameters are provided in Section 5 and a numerical simulation study is conducted to assess the performance these estimates. The flexibility of the OLxF distribution is proved empirically by two real data applications in Section 6. We provide some conclusions in Section 7.

\section{The OLxF distribution}

In this section, we define the OLxF distribution. By inserting the CDF (1) into equation (3), we obtain the CDF of OLxF distribution (for $x>0$ )

$$
F(x ; \alpha, \beta, a, b)=1-\beta^{\alpha}\left\{\beta+\frac{\exp \left[-\left(\frac{a}{x}\right)^{b}\right]}{1-\exp \left[-\left(\frac{a}{x}\right)^{b}\right]}\right\}^{-\alpha}
$$

The PDF corresponding to (5) is 


$$
f(x ; \alpha, \beta, a, b)=\alpha \beta^{\alpha} \frac{b a^{b} x^{-b-1} \exp \left[-\left(\frac{a}{x}\right)^{b}\right]}{\left\{1-\exp \left[-\left(\frac{a}{x}\right)^{b}\right]\right\}^{2}}\left\{\beta+\frac{\exp \left[-\left(\frac{a}{x}\right)^{b}\right]}{1-\exp \left[-\left(\frac{a}{x}\right)^{b}\right]}\right\}^{-\alpha-1},
$$

where $a>0$ is a scale parameter and $\beta>0, a>0$ and $b>0$ are shape parameters representing the different patterns of the OLxF distribution. Henceforth, a random variable $X$ having $\operatorname{PDF}(6)$ is denoted by $X \sim \operatorname{OLxF}(\alpha, \beta, a, b)$.

The HRF of $X$ is given by

$$
h(x ; \alpha, \beta, a, b)=\frac{\alpha b a^{b} x^{-b-1} \exp \left[-\left(\frac{a}{x}\right)^{b}\right]}{\left\{1-\exp \left[-\left(\frac{a}{x}\right)^{b}\right]\right\}^{2}\left\{\beta+\frac{\exp \left[-\left(\frac{a}{x}\right)^{b}\right]}{1-\exp \left[-\left(\frac{a}{x}\right)^{b}\right]}\right\}}
$$

The quantile function $(\mathrm{QF})$ of the OLxF model follows as

$$
Q(u)=a\left\{\log \frac{1+\beta(1-u)^{-1 / \alpha}-\beta}{\beta(1-u)^{-1 / \alpha}-\beta}\right\}^{-1 / b}, 0 \leq u \leq 1,
$$

where $U \sim U(0,1)$ is a uniform random number.

Figure 1 shows some plots of the $\mathrm{OLxF}$ density for selected values of $\alpha, \beta, a$ and $b$. The plots of the PDF indicate that the OLxF distribution can be reversed-J shaped, left-skewed or right-skewed. The plots of the HRF of the OLxF model are given in Figure 2 which reveals that the HRF can be increasing, decreasing or unimodal.

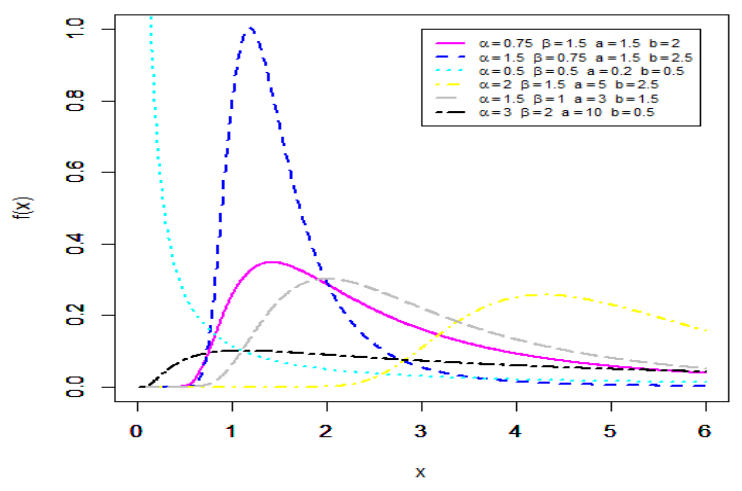

Figure 1: The PDF plots of the OLxF distribution

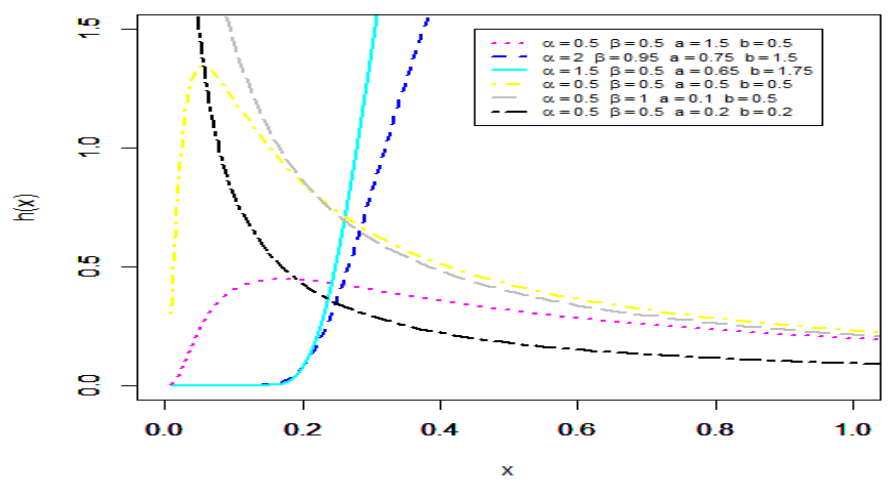

Figure 2: The HRF plots of the OLxF distribution 


\section{Linear representation}

In this section, we provide a useful expansion for the OLxF PDF in terms of Fréchet densities.

Using the following two power series given by

$$
(1-d z)^{-q}=\sum_{k=0}^{\infty}\left(\begin{array}{c}
-q \\
k
\end{array}\right) d^{k} z^{k}
$$

and

$$
(1-z)^{-q}=\sum_{j=0}^{\infty}(-1)^{j}\left(\begin{array}{c}
-q \\
j
\end{array}\right) z^{j}
$$

Cordeiro et al. (2019) provided a useful representation of the PDF of the OLx-G family as

$$
f(x)=\sum_{k, j=0}^{\infty} v_{k, j} h_{k+j+1}(x),
$$

where

$$
v_{k, j}=\frac{(-1)^{j} \alpha}{(k+j+1) \beta^{k+1}}\left(\begin{array}{c}
-\alpha-1 \\
k
\end{array}\right)\left(\begin{array}{c}
-k-2 \\
j
\end{array}\right)
$$

and $h_{k+j+1}(x)=(k+j+1) g(x) G(x)^{k+j}$ denotes the exponentiated-G (Exp-G) class density with power parameter $k+j+1>0$. Then, the PDF of the OLx-G class can be expressed as a double linear combination of Exp-G densities.

Using the CDF and PDF in (1) and (2) in Equation (8), we can write

$$
f(x)=\sum_{k, j=0}^{\infty} v_{k, j}(k+j+1) b a^{b} x^{-b-1} \exp \left[-(k+j+1)\left(\frac{a}{x}\right)^{b}\right],
$$

Hence, the PDF of the OLxF model can be expressed as

$$
f(x)=\sum_{k, j=0}^{\infty} v_{k, j} g_{k+j+1}(x),
$$

and $g_{k+j+1}(x)$ is the PDF of the Fréchet distribution with shape parameter $b$ and scale parameter $a(k+j+1)^{1 / b}$. Equation (9) reveals that the OLxF density can be written as a linear combination of Fréchet densities. Then, several of its properties can be obtained from those of the Fréchet distribution and Equation (9).

Let $Y$ be a random variable having the Fréchet distribution (1) with parameters $a$ and $b$. For $r<b$, the $n$th ordinary and incomplete moments of $Y$ are given by

$$
\mu_{n}^{\prime}=a^{n} \Gamma(1-n / b) \text { and } \varphi_{n, Y}(t)=a^{n} \gamma\left(1-n / b,(a / t)^{b}\right),
$$

respectively, where $\Gamma(\mathrm{s})=\int_{0}^{\infty} w^{s-1} e^{-w} d w$ is the complete gamma function and $\gamma(s, z)=\int_{0}^{z} w^{s-1} e^{-w} d w$ is the lower incomplete gamma function.

\section{The properties of the OLxF distribution}

In this section, we derive some mathematical properties of the OLxF distribution including ordinary and incomplete moments, moment generating function and order statistics.

\subsection{Ordinary and incomplete moments}


The $n$th ordinary moment of $X$ is given by

$$
\mu_{n}^{\prime}=E\left(X^{n}\right)=\sum_{k, j=0}^{\infty} v_{k, j} \int_{0}^{\infty} x^{n} g_{k+j+1}(x) d x
$$

For $n<b$, we obtain

$$
\mu_{n}^{\prime}=\sum_{k, j=0}^{\infty} v_{k, j} a^{n}(k+j+1)^{n / b} \Gamma(1-n / b) .
$$

Using Equation (10), we have the mean of $X$ with $n=1$.

The skewness and kurtosis measures can be evaluated from the ordinary moments using well-known relationships.

The $n$th incomplete moment of the OLxF distribution is defined by

$$
\varphi_{n}(t)=\int_{0}^{t} x^{n} f(x) d x
$$

Using Equation (9), we can write

$$
\varphi_{n}(t)=\sum_{k, j=0}^{\infty} v_{k, j} \int_{0}^{t} x^{n} g_{k+j+1}(x)
$$

Then, we have (for $n<b$ ),

$$
\varphi_{n}(t)=\sum_{k, j=0}^{\infty} v_{k, j} a^{n}(k+j+1)^{\frac{n}{b}} \gamma\left(1-n / b,(k+j+1)\left(\frac{a}{t}\right)^{b}\right) .
$$

The first incomplete moment, $\varphi_{1}(t)$, follows from the above equation with $n=1$. $\varphi_{1}(t)$ has an important application related to the Bonferroni and Lorenz curves. These curves are very useful in economics, demography, insurance, engineering and medicine.

Another application of $\varphi_{1}(t)$ is related to the mean residual life and the mean inactivity time defined by $m_{1}(t)=$ $\left[1-\varphi_{1}(t)\right] / R(t)-t$ and $M_{1}(t)=t-\varphi_{1}(t) / F(t)$, respectively.

\subsection{Moment generating function}

Afify et al. (2016) derived the moment generating function (MGF) of the Fréchet distribution, $M(t ; a, b)$, given by (1) and (2).

By setting $w=x^{-1}$, we can write

$$
M(t ; a, b)=b a^{b} \int_{0}^{\infty} \exp \left(\frac{t}{w}\right) w^{b-1} \exp \left[-(a w)^{b}\right] d w .
$$

Using the exponential series for the first exponential

$$
M(t ; a, b)=\mathrm{b} a^{b} \sum_{m=0}^{\infty} \frac{t^{m}}{m !} \int_{0}^{\infty} w^{\beta-m-1} \exp \left[-(a w)^{b}\right] d w .
$$

Calculating the integral, we obtain

$$
M(t ; a, b)=\sum_{m=0}^{\infty} \frac{a^{m} t^{m}}{m !} \Gamma\left(\frac{b-m}{b}\right) .
$$


Consider the Wright generalized hypergeometric function defined by

$$
{ }_{p} \Psi_{q}\left[\begin{array}{l}
\left(\alpha_{1}, A_{1}\right), \ldots,\left(\alpha_{p}, A_{p}\right) \\
\left(\beta_{1}, B_{1}\right), \ldots,\left(\beta_{q}, B_{q}\right)
\end{array} ; x\right]=\sum_{n=0}^{\infty} \frac{\prod_{j=1}^{p} \Gamma\left(\alpha_{j}+A_{j} n\right)}{\prod_{j=1}^{q} \Gamma\left(\beta_{j}+B_{j} n\right)} \frac{x^{n}}{n !} .
$$

Hence, we can write $M(t ; a, b)$ as

$$
M(t ; a, b)={ }_{1} \Psi_{0}\left[\left(1,-b^{-1}\right) ; a t\right] .
$$

Using Equations (9) and (11), the MGF of $X, M(t)$, reduces to

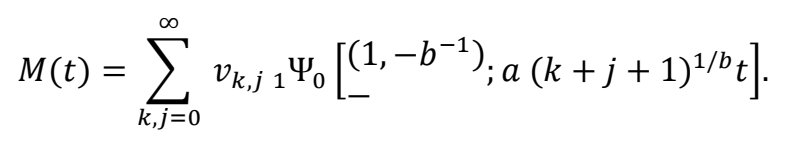

\subsection{Order statistics}

Let $X_{1}, \ldots, X_{n}$ be a random sample of size $n$ from the OLxF distribution and $X_{(1)}, \ldots, X_{(n)}$ be the corresponding order statistics. Then, the PDF of the $i$ th order statistic $X_{i: n}, f_{i: n}(x)$, is defined by

$$
\begin{aligned}
f_{i: n}(x)= & \frac{n !}{(i-1) !(n-i) !} f(x) F(x)^{i-1}[1-F(x)]^{n-i} \\
& =\frac{n !}{(i-1) !(n-i) !} f(x) \sum_{j=0}^{n-i}(-1)^{j}\left(\begin{array}{c}
n-i \\
j
\end{array}\right) F(x)^{j+i-1}
\end{aligned}
$$

Cordeiro et al. (2019) derived a simple formula for the $i$ th order statistic of the OLx-G family. The PDF of the $i$ th order statistic can be expressed as

$$
f_{i: n}(x)=\sum_{r, s=0}^{\infty} d_{r, s}(s+r+1) g(x) G(x)^{s+r},
$$

where

$$
d_{r, \mathrm{~s}}=\sum_{j=0}^{n-i} \sum_{k=0}^{j+i-1} \frac{(-1)^{k+s} n ! \alpha \beta^{-r-1}}{(i-1) !(n-i) !}\left(\begin{array}{c}
n-i \\
j
\end{array}\right)\left(\begin{array}{c}
-r-2 \\
s
\end{array}\right)\left(\begin{array}{c}
j+i-1 \\
k
\end{array}\right)\left(\begin{array}{c}
-(k+1) \alpha-1 \\
r
\end{array}\right) .
$$

Taking $G(x)$ and $g(x)$ in Equation (12) to be the CDF and PDF of the Fréchet distribution, then Equation (12) can be rewritten as

$$
f_{i: n}(x)=\sum_{r, s=0}^{\infty} d_{r, \mathrm{~s}}(s+r+1) b a^{b} x^{-b-1} \exp \left[-(s+r+1)\left(\frac{a}{x}\right)^{b}\right] .
$$

The last equation reduces to

$$
f_{i: n}(x)=\sum_{r, s=0}^{\infty} d_{r, s} g_{(s+r+1)}(x),
$$

where $g_{(s+r+1)}(x)$ denotes the density function of the Fréchet model with shape parameters $b$ and scale parameter $a(s+r+1)^{1 / b}$. Hence, the PDF of the OLxF order statistics is a linear mixture of Fréchet PDFs. Based on Equation (13), we can easily derive the $s$ th moment of $X_{i: n}$ is given (for $q<b$ ) by

$$
E\left(X_{i: n}^{q}\right)=\sum_{r, s=0}^{\infty} d_{r, s} a^{q}(s+r+1)^{\frac{q}{b}} \Gamma\left(1-\frac{q}{b}\right) .
$$




\section{Estimation and simulation}

In this section, we provide the estimation of the OLxF parameters from complete samples only by maximum likelihood estimation method. We investigate the MLEs of the parameters of the $\operatorname{OLxF}(\alpha, \beta, a, b)$ model. Let $x_{1}, \ldots, x_{n}$ be a random sample from the $\mathrm{OLxF}$ model with parameter vector $v=(\alpha, \beta, a, b)^{T}$.

The log-likelihood function for $v, \ell=\ell(v)$, is

$$
\begin{aligned}
\ell= & n(\log \alpha+\alpha \log \beta+\log b+b \log a)-(b+1) \sum_{i=1}^{n} \log x_{i}-\sum_{i=1}^{n}\left(\frac{a}{x_{i}}\right)^{b}-2 \sum_{i=1}^{n} \log \left(1-D_{i}\right) \\
& -(\alpha+1) \sum_{i=1}^{n} \log \left[\beta+D_{i} /\left(1-D_{i}\right)\right],
\end{aligned}
$$

where $D_{i}=\exp \left[-\left(\frac{a}{x_{i}}\right)^{b}\right]$

The above equation can be maximized either directly by using the SAS, R, Ox program or by solving the nonlinear likelihood equations obtained by differentiating it.

The score vector elements, $\mathbf{U}(v)=\frac{\partial \ell}{\partial v}=\left(\frac{\partial \ell}{\partial \alpha}, \frac{\partial \ell}{\partial \beta}, \frac{\partial \ell}{\partial a}, \frac{\partial \ell}{\partial b}\right)^{T}$, are given by

$$
\begin{gathered}
\frac{\partial \ell}{\partial \alpha}=\frac{n}{\alpha}+n \log \beta-\sum_{i=1}^{n} \log \left(\beta+\frac{D_{i}}{1-D_{i}}\right) \\
\frac{\partial \ell}{\partial \beta}=\frac{n \alpha}{\beta}-(\alpha+1) \sum_{i=1}^{n} \frac{1}{\beta+D_{i} /\left(1-D_{i}\right)^{\prime}} \\
\frac{\partial \ell}{\partial a}=\frac{n b}{a}+(\alpha+1) \sum_{i=1}^{n} \frac{b a^{b-1} x_{i}^{-b} D_{i}\left(D_{i}\left(1-D_{i}\right)+1\right)}{\left(1-D_{i}\right)^{2}\left[\beta+D_{i} /\left(1-D_{i}\right)\right]}-b a^{b-1} \sum_{i=1}^{n} x_{i}^{-b}-2 \sum_{i=1}^{n} \frac{b a^{b-1} x_{i}^{-b} D_{i}}{1-D_{i}}
\end{gathered}
$$

and

$$
\begin{aligned}
\frac{\partial \ell}{\partial b}=\frac{n}{b}+n & \log a-\sum_{i=1}^{n} \log x_{i}+(\alpha+1) \sum_{i=1}^{n} \frac{D_{i}\left(\frac{a}{x_{i}}\right)^{b} \log \left(\frac{a}{x_{i}}\right)\left(D_{i}\left(1-D_{i}\right)+1\right)}{\left(1-D_{i}\right)^{2}\left[\beta+D_{i} /\left(1-D_{i}\right)\right]}-\sum_{i=1}^{n}\left(\frac{a}{x_{i}}\right)^{b} \log \left(\frac{a}{x_{i}}\right) \\
& -2 \sum_{i=1}^{n} \frac{\left(\frac{a}{x_{i}}\right)^{b} D_{i} \log \left(\frac{a}{x_{i}}\right)}{1-D_{i}} .
\end{aligned}
$$

We can obtain the estimates of the unknown parameters by setting the score vector to zero, $\mathbf{U}(\hat{v})=\mathbf{0}$. By solving these equations simultaneously gives the maximum likelihood estimates $\hat{\alpha}, \widehat{\beta}$ and $\hat{\theta}$. These estimates can be obtained numerically using iterative techniques such as the Newton-Raphson algorithm.

Now we study the performance and behavior of maximum likelihood estimators (MLEs) of OLxF parameters by generating 10,000 samples of the OLxF distribution using the QF in Equation (7). We generate different samples of sizes $n=30,50,80,200$ and 400, for different combination of the OLxF parameters $\alpha, \beta, a$ and $b$. 
For each parameters combination and each sample, we calculate the empirical mean the estimates (EMs), mean square error of the estimates (MSE), empirical absolute bias (AB) and mean relative estimate (MRE). The MSE and AB are calculated as follows

$$
\begin{aligned}
M S E_{\hat{\boldsymbol{\theta}}} & =\frac{1}{N} \sum_{i=1}^{N}\left(\boldsymbol{\theta}-\widehat{\boldsymbol{\theta}}_{i}\right)^{2}, \\
A B_{\hat{\boldsymbol{\theta}}} & =\frac{1}{N} \sum_{i=1}^{N}\left|\boldsymbol{\theta}-\widehat{\boldsymbol{\theta}}_{i}\right|
\end{aligned}
$$

and

$$
M R E_{\hat{\boldsymbol{\theta}}}=\frac{1}{N} \sum_{i=1}^{N} \frac{\left|\boldsymbol{\theta}-\hat{\boldsymbol{\theta}}_{i}\right|}{\boldsymbol{\theta}}
$$

where $\widehat{\boldsymbol{\theta}}_{i}=(\alpha, \beta, a, b)$. The results of MLEs are obtained using the R software (version 3.3.1). The empirical results are reported in Tables 1-5. The results in Tables 1-5 illustrate that the empirical means approach the true parameter values when the sample size increases. Further, it can be observed that the values the MSEs, ABs and MREs decrease, as expected, in all cases as sample size increases. Therefore, the MLE method works very well to estimate the OLxF parameters.

\section{Application of OLF model}

In this section, we provide two applications of the OLxF distribution using two real data sets. The first data set refers to the exceedances of flood peaks (in $\mathrm{m}^{3} / \mathrm{s}$ ) of the Wheaton River near Carcross in Yukon Territory, Canada. The data consist of 72 exceedances for the years 1958-1984, rounded to one decimal place (Choulakian and Stephens, 2001).

Table 1: The EMs, MSEs, Abs and MREs for the OLxF distribution

\begin{tabular}{ccccc}
\hline Parameters & \multicolumn{4}{c}{$n=30$} \\
\hline$\alpha=2.0$ & EMs & MSEs & ABs & MREs \\
\hline$\beta=2.4$ & 1.99174 & 0.00176 & 0.00845 & 0.00422 \\
$a=2.5$ & 2.40561 & 0.00104 & 0.00564 & 0.00235 \\
$b=3.1$ & 3.48439 & 0.00198 & 0.01561 & 0.00624 \\
$\alpha=2.0$ & 1.99331 & 0.00126 & 0.00698 & 0.00349 \\
$\beta=1.5$ & 1.50705 & 0.00132 & 0.00711 & 0.00474 \\
$a=2.5$ & 2.48439 & 0.00197 & 0.01561 & 0.00624 \\
$b=3.1$ & 3.10760 & 0.00181 & 0.00816 & 0.00263 \\
\hline$\alpha=0.75$ & 0.74852 & 0.00021 & 0.00165 & 0.00220 \\
$\beta=1.50$ & 1.50031 & 0.00008 & 0.00081 & 0.00054 \\
$a=1.50$ & 1.49634 & 0.00078 & 0.00366 & 0.00244 \\
$b=0.75$ & 0.75322 & 0.00043 & 0.00322 & 0.00430 \\
\hline$\alpha=0.75$ & 0.74949 & 0.00009 & 0.00052 & 0.00069 \\
$\beta=1.50$ & 1.50061 & 0.00017 & 0.00061 & 0.00041 \\
$a=2.50$ & 2.49848 & 0.00051 & 0.00152 & 0.00061 \\
$b=1.75$ & 1.75119 & 0.00044 & 0.00119 & 0.00068 \\
\hline
\end{tabular}




\begin{tabular}{lllll}
\hline$\alpha=0.75$ & 0.74955 & 0.00003 & 0.00050 & 0.00067 \\
$\beta=0.50$ & 0.50067 & 0.00009 & 0.00068 & 0.00136 \\
$a=1.50$ & 1.49798 & 0.00031 & 0.00202 & 0.00135 \\
$b=1.75$ & 1.75137 & 0.00027 & 0.00137 & 0.00078 \\
\hline$\alpha=2.00$ & 1.99960 & 0.00001 & 0.00050 & 0.00025 \\
$\beta=0.50$ & 0.50132 & 0.00009 & 0.00141 & 0.00282 \\
$a=0.35$ & 0.34678 & 0.00005 & 0.00322 & 0.00921 \\
$b=1.75$ & 1.75089 & 0.00006 & 0.00117 & 0.00067 \\
\hline$\alpha=0.65$ & 0.64863 & 0.00006 & 0.00159 & 0.00245 \\
$\beta=2.40$ & 2.40002 & 0.00000 & 0.00032 & 0.00013 \\
$a=0.35$ & 0.34800 & 0.00008 & 0.00202 & 0.00576 \\
$b=0.25$ & 0.25293 & 0.00006 & 0.00293 & 0.01173 \\
\hline$\alpha=0.65$ & 0.64773 & 0.00026 & 0.00282 & 0.00434 \\
$\beta=2.50$ & 2.49943 & 0.00013 & 0.00110 & 0.00044 \\
$a=4.50$ & 4.49884 & 0.00013 & 0.00116 & 0.00026 \\
$b=0.45$ & 0.45482 & 0.00044 & 0.00482 & 0.01072 \\
\hline
\end{tabular}

Table 2: The EMs, MSEs, Abs and MREs for the OLxF distribution

\begin{tabular}{ccccc}
\hline Parameters & \multicolumn{4}{c}{$n=50$} \\
\hline & EMs & MSEs & ABs & MREs \\
\hline$\alpha=2.0$ & 1.99022 & 0.00185 & 0.00999 & 0.00499 \\
$\beta=2.4$ & 2.40638 & 0.00080 & 0.00640 & 0.00267 \\
$a=2.5$ & 2.47949 & 0.00241 & 0.02051 & 0.00820 \\
$b=3.1$ & 3.10923 & 0.00147 & 0.00984 & 0.00317 \\
\hline$\alpha=2.0$ & 1.99236 & 0.00122 & 0.00792 & 0.00396 \\
$\beta=1.5$ & 1.50809 & 0.00141 & 0.00815 & 0.00543 \\
$a=2.5$ & 2.48031 & 0.00227 & 0.01969 & 0.00788 \\
$b=3.1$ & 3.10864 & 0.00175 & 0.00923 & 0.00298 \\
\hline$\alpha=0.75$ & 0.74769 & 0.00023 & 0.00254 & 0.00339 \\
$\beta=1.50$ & 1.50032 & 0.00005 & 0.00101 & 0.00068 \\
$a=1.50$ & 1.49442 & 0.00089 & 0.00558 & 0.00372 \\
$b=0.75$ & 0.75512 & 0.00052 & 0.00512 & 0.00683 \\
\hline$\alpha=0.75$ & 0.74926 & 0.00010 & 0.00076 & 0.00101 \\
$\beta=1.50$ & 1.50075 & 0.00015 & 0.00075 & 0.00050 \\
$a=2.50$ & 2.49780 & 0.00060 & 0.00220 & 0.00088 \\
$b=1.75$ & 1.75177 & 0.00052 & 0.00177 & 0.00101 \\
\hline$\alpha=0.75$ & 0.74899 & 0.00010 & 0.00107 & 0.00143 \\
$\beta=0.50$ & 0.50141 & 0.00021 & 0.00143 & 0.00286 \\
$a=1.50$ & 1.49613 & 0.00066 & 0.00387 & 0.00258 \\
$b=1.75$ & 1.75284 & 0.00073 & 0.00284 & 0.00162 \\
\hline$\alpha=2.00$ & 1.99952 & 0.00002 & 0.00056 & 0.00028 \\
$\beta=0.50$ & 0.50163 & 0.00016 & 0.00169 & 0.00337 \\
$a=0.35$ & 0.34623 & 0.00006 & 0.00377 & 0.01076 \\
$b=1.75$ & 1.75103 & 0.00009 & 0.00125 & 0.00071 \\
\hline$\alpha=0.65$ & 0.64796 & 0.00009 & 0.00219 & 0.00337 \\
$\beta=2.40$ & 2.40012 & 0.00000 & 0.00035 & 0.00015 \\
$a=0.35$ & 0.34771 & 0.00009 & 0.00230 & 0.00657 \\
$b=0.25$ & 0.25374 & 0.00008 & 0.00374 & 0.01497 \\
\hline$\alpha=0.65$ & 0.64675 & 0.00024 & 0.00360 & 0.00554 \\
$\beta=2.50$ & 2.49989 & 0.00004 & 0.00083 & 0.00033 \\
$a=4.50$ & 4.49900 & 0.00005 & 0.00100 & 0.00022 \\
$b=0.45$ & 0.45585 & 0.00037 & 0.00585 & 0.01301 \\
\hline & & & &
\end{tabular}


Table 3: The EMs, MSEs, Abs and MREs for the OLxF distribution

\begin{tabular}{|c|c|c|c|c|}
\hline \multirow[t]{2}{*}{ Parameters } & \multicolumn{4}{|c|}{$n=80$} \\
\hline & EMs & MSEs & $\mathrm{ABs}$ & MREs \\
\hline$\alpha=2.0$ & 1.98837 & 0.00221 & 0.01184 & 0.00592 \\
\hline$\beta=2.4$ & 2.40756 & 0.00093 & 0.00758 & 0.00316 \\
\hline$a=2.5$ & 2.47394 & 0.00295 & 0.02606 & 0.01042 \\
\hline$b=3.1$ & 3.11136 & 0.00217 & 0.01205 & 0.00389 \\
\hline$\alpha=2.0$ & 1.99139 & 0.00141 & 0.00890 & 0.00445 \\
\hline$\beta=1.5$ & 1.50943 & 0.00173 & 0.00948 & 0.00632 \\
\hline$a=2.5$ & 2.47648 & 0.00269 & 0.02352 & 0.00941 \\
\hline$b=3.1$ & 3.11052 & 0.00237 & 0.01102 & 0.00356 \\
\hline$\alpha=0.75$ & 0.74620 & 0.00044 & 0.00407 & 0.00543 \\
\hline$\beta=1.50$ & 1.50045 & 0.00005 & 0.00129 & 0.00086 \\
\hline$a=1.50$ & 1.49126 & 0.00167 & 0.00874 & 0.00583 \\
\hline$b=0.75$ & 0.75771 & 0.00087 & 0.00771 & 0.01029 \\
\hline$\alpha=0.75$ & 0.74886 & 0.00014 & 0.00116 & 0.00155 \\
\hline$\beta=1.50$ & 1.50106 & 0.00017 & 0.00106 & 0.00070 \\
\hline$a=2.50$ & 2.49652 & 0.00085 & 0.00348 & 0.00139 \\
\hline$b=1.75$ & 1.75275 & 0.00066 & 0.00275 & 0.00157 \\
\hline$\alpha=0.75$ & 0.74831 & 0.00014 & 0.00179 & 0.00238 \\
\hline$\beta=0.50$ & 0.50251 & 0.00035 & 0.00253 & 0.00507 \\
\hline$a=1.50$ & 1.49316 & 0.00116 & 0.00684 & 0.00456 \\
\hline$b=1.75$ & 1.75464 & 0.00098 & 0.00464 & 0.00265 \\
\hline$\alpha=2.00$ & 1.99951 & 0.00001 & 0.00054 & 0.00027 \\
\hline$\beta=0.50$ & 0.50164 & 0.00011 & 0.00167 & 0.00335 \\
\hline$a=0.35$ & 0.34574 & 0.00007 & 0.00426 & 0.01218 \\
\hline$b=1.75$ & 1.75111 & 0.00007 & 0.00125 & 0.00071 \\
\hline$\alpha=0.65$ & 0.64715 & 0.00013 & 0.00297 & 0.00456 \\
\hline$\beta=2.40$ & 2.40018 & 0.00000 & 0.00038 & 0.00016 \\
\hline$a=0.35$ & 0.34719 & 0.00012 & 0.00282 & 0.00806 \\
\hline$b=0.25$ & 0.25478 & 0.00011 & 0.00478 & 0.01911 \\
\hline$\alpha=0.65$ & 0.64602 & 0.00026 & 0.00424 & 0.00653 \\
\hline$\beta=2.50$ & 2.50002 & 0.00002 & 0.00076 & 0.00030 \\
\hline$a=4.50$ & 4.49893 & 0.00010 & 0.00107 & 0.00024 \\
\hline$b=0.45$ & 0.45703 & 0.00037 & 0.00703 & 0.01563 \\
\hline
\end{tabular}

Table 4: The EMs, MSEs, Abs and MREs for the OLxF distribution

\begin{tabular}{ccccc}
\hline Parameters & \multicolumn{4}{c}{$n=200$} \\
\hline$\alpha=2.0$ & EMs & MSEs & ABs & MREs \\
\hline$\beta=2.4$ & 1.99145 & 0.00150 & 0.00870 & 0.00435 \\
$a=2.5$ & 2.40576 & 0.00075 & 0.00577 & 0.00241 \\
$b=3.1$ & 2.47394 & 0.00252 & 0.02606 & 0.01042 \\
$\alpha=2.0$ & 3.11051 & 0.00174 & 0.01065 & 0.00344 \\
$\beta=1.5$ & 1.99372 & 0.00077 & 0.00647 & 0.00323 \\
$a=2.5$ & 1.50684 & 0.00089 & 0.00688 & 0.00459 \\
$b=3.1$ & 2.47565 & 0.00224 & 0.02435 & 0.00974 \\
$\alpha=0.75$ & 3.10866 & 0.00123 & 0.00882 & 0.00285 \\
$\beta=1.50$ & 0.74402 & 0.00055 & 0.00626 & 0.00835 \\
$a=1.50$ & 1.50065 & 0.00003 & 0.00143 & 0.00095 \\
$b=0.75$ & 1.48828 & 0.00168 & 0.01172 & 0.00782 \\
\hline
\end{tabular}




\begin{tabular}{lllll}
\hline$\alpha=0.75$ & 0.74736 & 0.00036 & 0.00265 & 0.00353 \\
$\beta=1.50$ & 1.50165 & 0.00019 & 0.00165 & 0.00110 \\
$a=2.50$ & 2.49287 & 0.00189 & 0.00713 & 0.00285 \\
$b=1.75$ & 1.75596 & 0.00161 & 0.00596 & 0.00340 \\
\hline$\alpha=0.75$ & 0.74712 & 0.00023 & 0.00307 & 0.00410 \\
$\beta=0.50$ & 0.50430 & 0.00063 & 0.00431 & 0.00863 \\
$a=1.50$ & 1.48742 & 0.00191 & 0.01258 & 0.00839 \\
$b=1.75$ & 1.75787 & 0.00158 & 0.00787 & 0.00450 \\
\hline$\alpha=2.00$ & 1.99972 & 0.00001 & 0.00029 & 0.00015 \\
$\beta=0.50$ & 0.50095 & 0.00010 & 0.00096 & 0.00192 \\
$a=0.35$ & 0.34610 & 0.00005 & 0.00390 & 0.01115 \\
$b=1.75$ & 1.75065 & 0.00007 & 0.00070 & 0.00040 \\
\hline$\alpha=0.65$ & 0.64703 & 0.00012 & 0.00302 & 0.00464 \\
$\beta=2.40$ & 2.40026 & 0.00000 & 0.00034 & 0.00014 \\
$a=0.35$ & 0.34757 & 0.00007 & 0.00243 & 0.00694 \\
$b=0.25$ & 0.25557 & 0.00012 & 0.00557 & 0.02228 \\
\hline$\alpha=0.65$ & 0.64525 & 0.00024 & 0.00488 & 0.00751 \\
$\beta=2.50$ & 2.50030 & 0.00000 & 0.00054 & 0.00022 \\
$a=4.50$ & 4.49921 & 0.00001 & 0.00079 & 0.00018 \\
$b=0.45$ & 0.45890 & 0.00037 & 0.00890 & 0.01978 \\
\hline
\end{tabular}

Table 5: The EMs, MSEs, Abs and MREs for the OLxF distribution

\begin{tabular}{lcccc}
\hline Parameters & \multicolumn{4}{c}{$n=400$} \\
\hline & EMs & MSEs & ABs & MREs \\
\hline$\alpha=2.0$ & 1.99690 & 0.00020 & 0.00319 & 0.00159 \\
$\beta=2.4$ & 2.40209 & 0.00010 & 0.00210 & 0.00088 \\
$a=2.5$ & 2.48089 & 0.00127 & 0.01911 & 0.00764 \\
$b=3.1$ & 3.10568 & 0.00030 & 0.00569 & 0.00183 \\
\hline$\alpha=2.0$ & 1.99759 & 0.00008 & 0.00250 & 0.00125 \\
$\beta=1.5$ & 1.50263 & 0.00009 & 0.00266 & 0.00177 \\
$a=2.5$ & 2.48176 & 0.00114 & 0.01824 & 0.00730 \\
$b=3.1$ & 3.10431 & 0.00013 & 0.00432 & 0.00139 \\
\hline$\alpha=0.75$ & 0.74417 & 0.00041 & 0.00604 & 0.00805 \\
$\beta=1.50$ & 1.50065 & 0.00001 & 0.00113 & 0.00075 \\
$a=1.50$ & 1.48932 & 0.00104 & 0.01068 & 0.00712 \\
$b=0.75$ & 0.76333 & 0.00095 & 0.01333 & 0.01778 \\
\hline$\alpha=0.75$ & 0.74673 & 0.00031 & 0.00328 & 0.00437 \\
$\beta=1.50$ & 1.50152 & 0.00009 & 0.00152 & 0.00101 \\
$a=2.50$ & 2.49040 & 0.00173 & 0.00960 & 0.00384 \\
$b=1.75$ & 1.75785 & 0.00141 & 0.00785 & 0.00449 \\
\hline$\alpha=0.75$ & 0.74654 & 0.00021 & 0.00364 & 0.00486 \\
$\beta=0.50$ & 0.50473 & 0.00042 & 0.00475 & 0.00949 \\
$a=1.50$ & 1.48370 & 0.00203 & 0.01630 & 0.01086 \\
$b=1.75$ & 1.75885 & 0.00121 & 0.00885 & 0.00506 \\
\hline$\alpha=2.00$ & 1.99991 & 0.00000 & 0.00010 & 0.00005 \\
$\beta=0.50$ & 0.50032 & 0.00000 & 0.00032 & 0.00065 \\
$a=0.35$ & 0.34676 & 0.00003 & 0.00324 & 0.00926 \\
$b=1.75$ & 1.75020 & 0.00000 & 0.00023 & 0.00013 \\
\hline$\alpha=0.65$ & 0.64788 & 0.00008 & 0.00215 & 0.00331 \\
$\beta=2.40$ & 2.40019 & 0.00000 & 0.00022 & 0.00009 \\
$a=0.35$ & 0.34824 & 0.00004 & 0.00176 & 0.00502 \\
$b=0.25$ & 0.25517 & 0.00009 & 0.00517 & 0.02067 \\
\hline & & & &
\end{tabular}




\begin{tabular}{lllll}
\hline$\alpha=0.65$ & 0.64582 & 0.00017 & 0.00425 & 0.00654 \\
$\beta=2.50$ & 2.50032 & 0.00000 & 0.00042 & 0.00017 \\
$a=4.50$ & 4.49936 & 0.00000 & 0.00064 & 0.00014 \\
$b=0.45$ & 0.45905 & 0.00032 & 0.00905 & 0.02011 \\
\hline
\end{tabular}

The second data set represents the remission times (in months) of 128 bladder cancer patients (Lee and Wang, 2003).

We compare the fits of the OLxF distribution with some important extensions of the Fréchet model namely, the Kumaraswamy Fréchet (KF),

exponentiated Fréchet $(\mathrm{EF})$, beta Fréchet $(\mathrm{BF})$, gamma extended Fréchet (GExF) and Fréchet $(\mathrm{F})$ distributions with corresponding PDFs (for $x>0$ ):

$\mathrm{KF}: f(x)=\alpha \beta b a^{b} x^{-(b+1)} \exp \left[-\alpha\left(\frac{a}{x}\right)^{b}\right]\left\{1-\exp \left[-\alpha\left(\frac{a}{x}\right)^{b}\right]\right\}^{\beta-1} ;$

$\mathrm{EF}: f(x)=\theta b a^{b} x^{-(b+1)} \exp \left[-\left(\frac{a}{x}\right)^{b}\right]\left\{1-\exp \left[-\left(\frac{a}{x}\right)^{b}\right]\right\}^{\theta-1}$;

$\mathrm{BF}: f(x)=\frac{b a^{b}}{B(\alpha, \beta)} x^{-(b+1)} \exp \left[-\alpha\left(\frac{a}{x}\right)^{b}\right]\left\{1-\exp \left[-\left(\frac{a}{x}\right)^{b}\right]\right\}^{\beta-1}$;

GExF: $f(x)=\frac{\alpha b a^{b}}{\Gamma(\beta)} x^{-(b+1)} \exp \left[-\left(\frac{a}{x}\right)^{b}\right]\left\{1-\exp \left[-\left(\frac{a}{x}\right)^{b}\right]\right\}^{\alpha-1}\left\{-\log \left\{1-\exp \left[-\left(\frac{a}{x}\right)^{b}\right]\right\}^{\alpha}\right\}^{\beta-1}$;

TEF: $f(x)=\alpha b a^{b} x^{-(b+1)} \exp \left[-\left(\frac{a}{x}\right)^{b}\right]\left\{1-\exp \left[-\left(\frac{a}{x}\right)^{b}\right]\right\}^{\alpha-1}\left(1+\beta-2 \beta\left\{1-\exp \left[-\left(\frac{a}{x}\right)^{b}\right]\right\}^{\alpha}\right)$.

The parameters of the above densities are all positive real numbers except for the TEF distribution for which $|\beta| \leq 1$.

We compare the fitted distributions using the following goodness-of-fit measures namely, the minus maximized loglikelihood $(-\widehat{\ell})$, Cramér-Von Mises $\left(W^{*}\right)$, Anderson-Darling $\left(A^{*}\right)$ statistics, Kolmogorov- Smirnov (KS) statistic and its p-value $(\mathrm{PV})$.

Tables 6 and 7 provide the values of $-\widehat{\ell}, W^{*}, A^{*} \mathrm{KS}, \mathrm{PV}$, the MLEs and their corresponding standard errors (in parentheses) for both data sets respectively. The plots of the fitted OLxF PDF, CDF, survival function (SS) and probability probability (PP) plots for both data sets are displayed in Figures 3 and 4, respectively.

Its noted, from Tables 1 and 2, that the OLxF model has the lowest values for the $-\hat{\ell}, W^{*}, A^{*}$ and KS statistics and largest value for the PV among all fitted models. Then, the OLxF model can be chosen as the best model for Wheaton river data. The HRF plots of the OLxF distribution for the two data sets are shown in Figure 5. One can see that the HRF of the OLxF model is unimodal for both data sets.

Table 6: Goodness-of-fit measures, MLEs and their SEs for Wheaton river data

\begin{tabular}{ccccccc}
\hline Model & $-\hat{\ell}$ & $\mathrm{W}^{*}$ & $\mathrm{~A}^{*}$ & $\mathrm{KS}(\mathrm{PV})$ & & Estimates \\
\hline OLxF & 251.192 & 0.1407 & 0.7783 & 0.0921 & $\alpha$ & $30.887(48.451)$ \\
& & & & $(0.5750)$ & $\beta$ & $571.56(848.28)$ \\
& & & & & $a$ & $0.3411(0.3147)$ \\
& & & & & $b$ & $0.8486(0.1079)$ \\
\hline $\mathrm{KF}$ & 252.365 & 0.1724 & 0.9515 & 0.1011 & $\alpha$ & $16.482(2.5773)$ \\
& & & & $(0.4540)$ & $\beta$ & $6334.1(11706)$ \\
& & & & & $a$ & $0.0158(0.0189)$ \\
& & & & & $b$ & $0.0969(0.0209)$ \\
\hline
\end{tabular}




\begin{tabular}{ccccccc}
\hline EF & 253.773 & 0.2079 & 1.1482 & 0.1205 & $a$ & $86107(8036.6)$ \\
& & & & $(0.2468)$ & $b$ & $0.1715(0.0065)$ \\
& & & & & $\theta$ & $110.29(29.949)$ \\
\hline BF & 256.935 & 0.2769 & 1.5583 & 0.1431 & $\alpha$ & $49.227(85.574)$ \\
& & & & $(0.1050)$ & $\beta$ & $90.127(117.85)$ \\
& & & & & $a$ & $10.139(74.482)$ \\
& & & & & $b$ & $0.0772(0.0512)$ \\
\hline GExF & 256.941 & 0.2771 & 1.5589 & 0.1431 & $\alpha$ & $96.955(133.88)$ \\
& & & & $(0.1047)$ & $\beta$ & $41.2559(74.551)$ \\
& & & & & $a$ & $12.854(94.924)$ \\
& & & & & $b$ & $0.0824(0.0559)$ \\
\hline F & 267.019 & 0.4426 & 2.5966 & 0.1532 & $a$ & $2.8790(0.5533)$ \\
& & & & $(0.0682)$ & $b$ & $0.6521(0.0538)$ \\
\hline
\end{tabular}

Table 7: Goodness-of-fit measures, MLEs and their SEs for cancer data

\begin{tabular}{ccccccc}
\hline Model & $-\hat{\ell}$ & $W^{*}$ & $A^{*}$ & $K S(P V)$ & & Estimates \\
\hline OLxF & 409.851 & 0.0238 & 0.1652 & 0.0406 & $\alpha$ & $2.1826(1.0476)$ \\
& & & & $(0.9843)$ & $\beta$ & $625.38(622.71)$ \\
& & & & & $a$ & $0.1221(0.0802)$ \\
& & & & & $b$ & $1.3856(0.1727)$ \\
\hline KF & 411.112 & 0.0532 & 0.3495 & 0.0507 & $\alpha$ & $3.1088(2.4272)$ \\
& & & & $(0.8972)$ & $\beta$ & $777.23(2205.7)$ \\
& & & & & $a$ & $1120.5(1943.1)$ \\
& & & & & $b$ & $0.1574(0.0651)$ \\
\hline EF & 412.429 & 0.0672 & 0.4664 & 0.0491 & $a$ & $1426.4(1440.19)$ \\
& & & & $(0.9175)$ & $b$ & $0.2624(0.0285)$ \\
& & & & & $\theta$ & $46.248(22.873)$ \\
\hline BF & 412.549 & 0.0690 & 0.4765 & 0.0554 & $\alpha$ & $0.6097(0.3226)$ \\
& & & & $(0.8268)$ & $\beta$ & $36.602(19.483)$ \\
& & & & & $a$ & $739.387(629.24)$ \\
& & & & & $b$ & $0.3224(0.0604)$ \\
\hline GExF & 416.108 & 0.1432 & 0.9597 & 0.0664 & $\alpha$ & $226.67(267.96)$ \\
& & & & $(0.6259)$ & $\beta$ & $91.939(133.94)$ \\
& & & & & $a$ & $22.271(119.19)$ \\
& & & & & $b$ & $0.0709(0.0413)$ \\
\hline F & 444.001 & 0.7443 & 4.5464 & 0.1408 & $a$ & $3.2582(0.4074)$ \\
& & & & $(0.0125)$ & $b$ & $0.7521(0.0424)$ \\
\hline
\end{tabular}



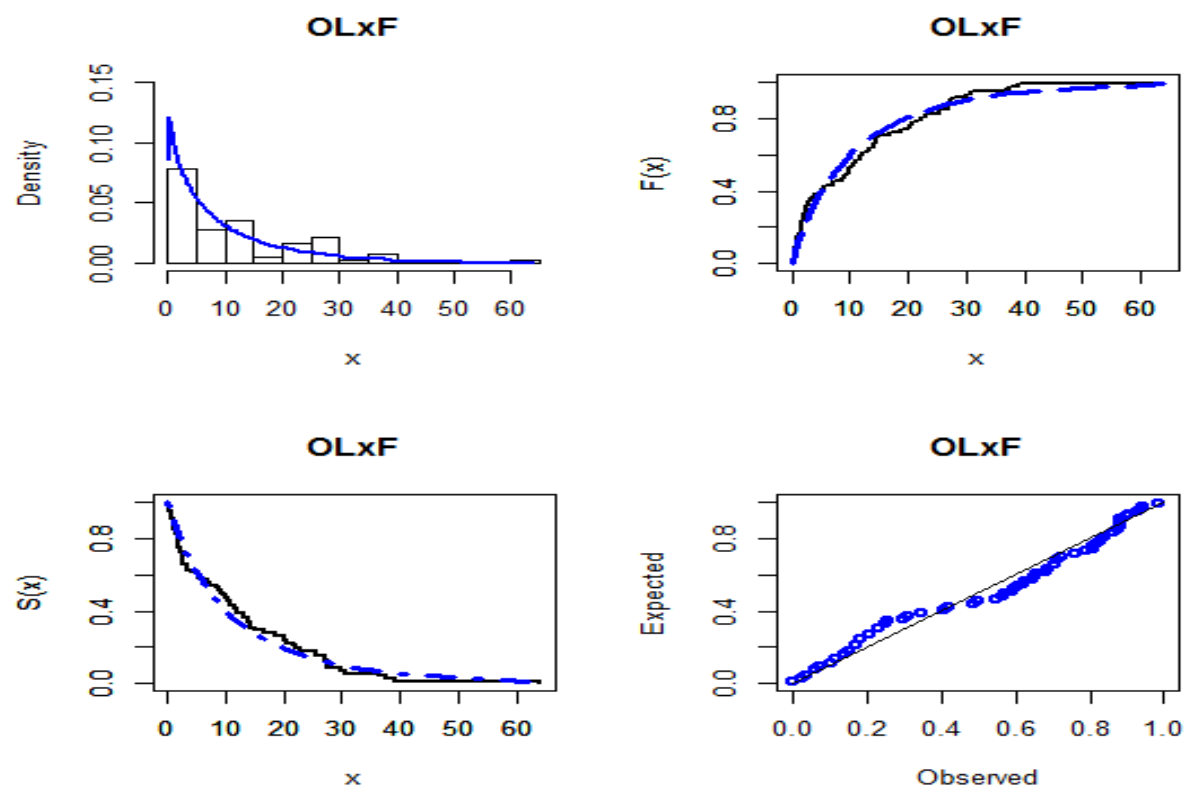

Figure 3: The fitted PDF, CDF, SF and PP plots of the OLxF model
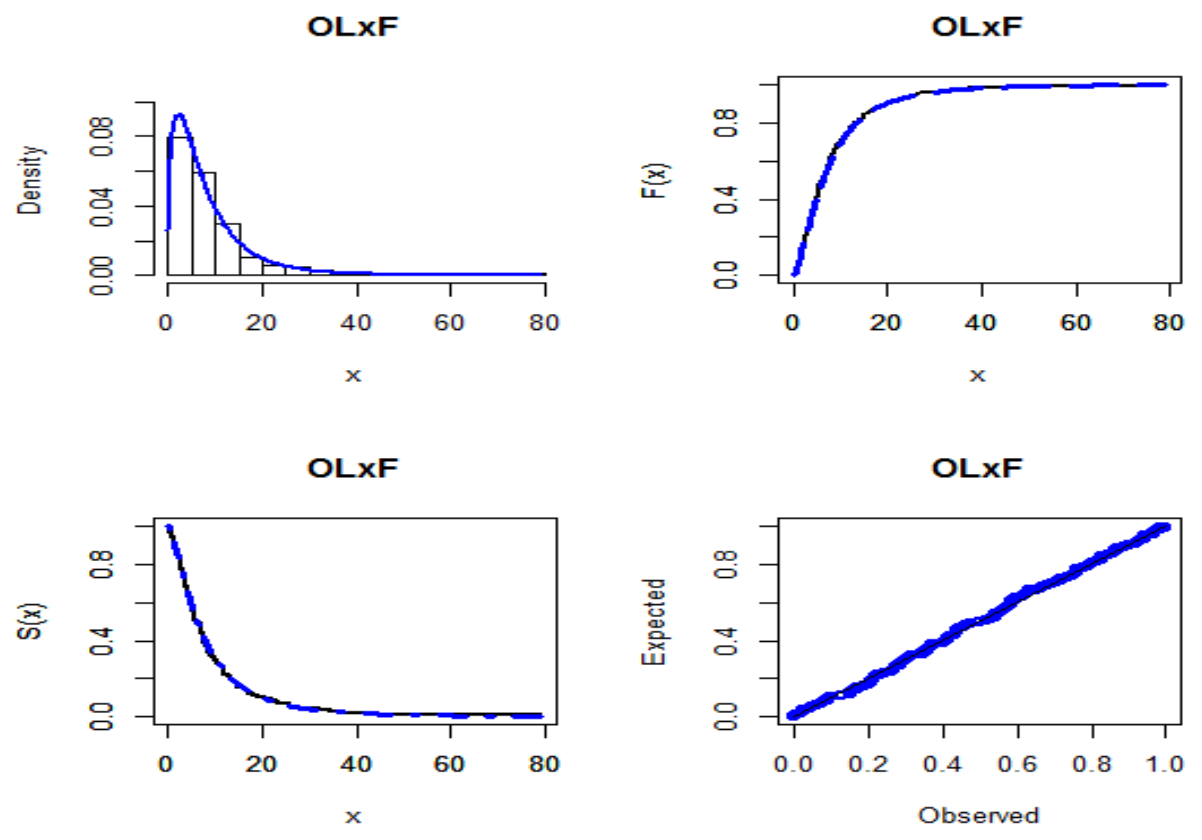

Figure 4: The fitted PDF, CDF, SF and PP plots of the OLxF model 

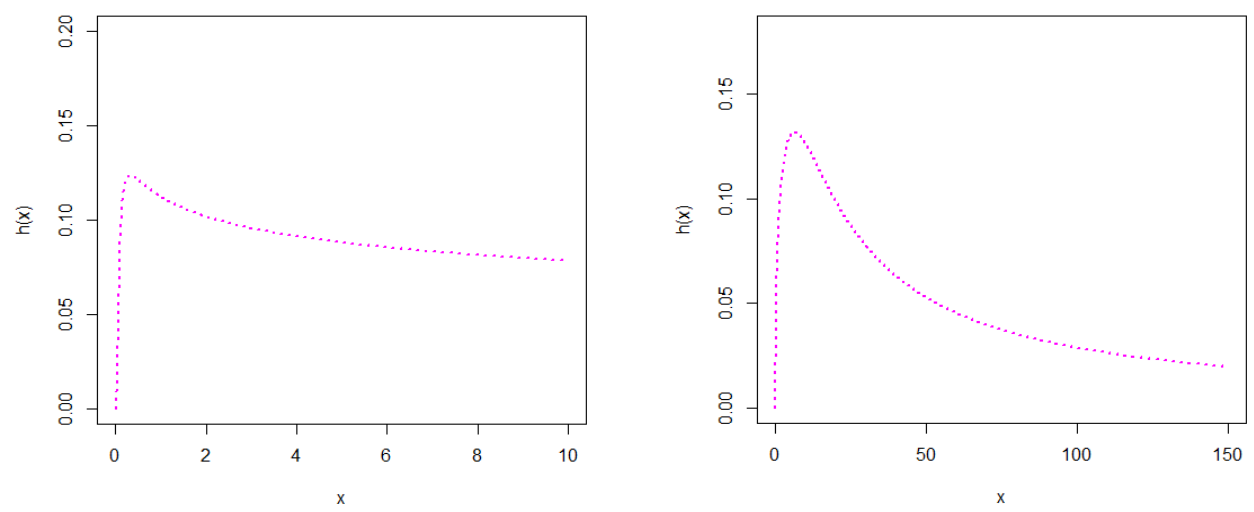

Figure 5: The HRF plot of the OLxF distribution

\section{Conclusions}

In this paper, we propose and study a new extension of the Fréchet model called the odd Lomax Fréchet (OLxF) distribution, which extends the Fréchet distribution. The OLxF PDF can be expressed as a linear mixture of Fréchet densities. We derive explicit expressions for its ordinary and incomplete moments, generating function and order statistics. The model parameters are estimated by maximum likelihood. Further, we conduct a numerical Monte Carlo simulation study which illustrates that the maximum likelihood approach performs very well in estimating the OLxF parameters. The proposed model provides better fits than some other well-known competitive models using two real data applications.

Acknowledgments: The authors would like to thank the editor and reviewers for their constructive comments and suggestions which greatly improved the final version of the manuscript.

\section{References}

1. Abouelmagd, T. H. M., Hamed, M. S., Afify, A. Z., Al-Mofleh, H. and Iqbal, Z. (2018). The Burr X Fréchet distribution with its properties and applications. Journal of Applied Probability and Statistics, $13,23-51$.

2. Afify, A. Z., Hamedani, G. G., Ghosh, I. and Mead, M. E. (2015). The transmuted Marshall-Olkin Fréchet distribution: properties and applications. International Journal of Statistics and Probability, 4, 132-184.

3. Afify, A. Z., Yousof, H. M., Cordeiro, G. M. Ortega, E. M. M. and Nofal, Z. M. (2016) The Weibull Fréchet distribution and its applications. Journal of Applied Statistics, 43, 2608-2626.

4. Choulakian, V. and Stephens, M. (2001). Goodness-of-Fit tests for the generalized Pareto distribution. Technometrics, 43, 478-484.

5. Cordeiro, G. M., Afify, A. Z., Ortega, E. M., Suzuki, A. K. and Mead, M. E. (2019). The odd Lomax generator of distributions: properties, estimation and applications. Journal of Computational and Applied Mathematics, 347, 222-237.

6. Fréchet M. (1924). Sur la loi des erreurs dIobservation. Bulletin de la Société Mathématique de Moscou, 33, 5-8.

7. Krishna, E., Jose, K. K., Alice, T. and Ristic, M. M. (2013). The Marshall-Olkin Fréchet distribution. Communications in Statistics-Theory and Methods, 42, 4091-4107.

8. Kotz, S. and Nadarajah, S. (2000). Extreme value distributions: theory and applications. Imperial College Press, London.

9. Lee, E. T. and Wang, J. W. (2003). Statistical Methods for Survival Data Analysis, 3rd edn. Wiley, New York. 
10. Mahmoud, M. R. and Mandouh, R. M. (2013). On the transmuted Fréchet distribution. Journal of Applied Sciences Research, 9, 5553-5561.

11. Mansour, M. M., Abd Elrazik, E. M., Altun, E., Afify, A. Z. and Iqbal, Z. (2018a). A new three-parameter Fréchet distribution: properties and applications. Pak. J. Statist, 34, 441-458.

12. Mansour, M. M., Aryal, G., Afify, A. Z. and Ahmad, M. (2018b). The kumaraswamy exponentiated Fréchet distribution. Pak. J. Statist, 34, 177-193.

13. Mead, M. E. and Abd-Eltawab A. R. (2014). A note on Kumaraswamy Fréchet distribution. Australian Journal of Basic and Applied Sciences, 8, 294-300.

14. Mead, M. E., Afify, A. Z., Hamedani, G. G. and Ghosh, I. (2017). The beta exponential Fréchet distribution with applications. Austrian Journal of Statistics, 46, 41-63.

15. Mubarak, M. (2011). Parameter estimation based on the Fréchet progressive type II censored data with binomial removals. Journal of Quality, Statistics and Reliability 2012 (2011).

16. Nadarajah, S. and Gupta, A. K. (2004). The beta Fréchet distribution. Far East Journal of Theoretical Statistics, 14, 15-24.

17. Nadarajah, S. and Kotz, S. (2003). The exponentiated Fréchet distribution. Interstat Electronic Journal, 1-7.

18. Ramos, P. L., Louzada, F., Ramos, E. and Dey, S. (2019). The Fréchet distribution: estimation and application-an overview. Journal of Statistics and Management Systems, 1-30.

19. Ramos, P. L., Nascimento, D. and Louzada, F. (2017). The Long Term Fréchet Distribution: Estimation, Properties and Its Application. Biom. Biostat. Int. J., 6, 357-362.

20. Silva, R. V. D., de Andrade, T. A., Maciel, D., Campos, R. P. and Cordeiro, G. M. (2013). A new lifetime model: the gamma extended Fréchet distribution. Journal of Statistical Theory and Applications, 12, 3954.

21. Tablada, C. J. and Cordeiro, G. M. (2017). The modified Fréchet distribution and its properties. Communications in Statistics-Theory and Methods, 46, 10617-10639. 